\title{
Constructing Definitive Screening Designs Using Cyclic Generators
}

\author{
NAM-KY NGUYEN ${ }^{1}$ AND STELLA STYLIANOU ${ }^{2}$ \\ ${ }^{1}$ International School \& Centre for High Performance Computing, Vietnam \\ National University, Hanoi, Vietnam \\ ${ }^{2}$ Department of Statistics \& Actuarial-Financial Mathematics, University of the \\ Aegean, Samos, Greece
}

\begin{abstract}
Jones and Nachtsheim (2011) propose a new class of computer-generated three-level screening designs called definitive screening designs (DSDs). These designs provide estimates of main effects that are unbiased by any second-order effect and require only one more than twice the number of factors. Stylianou (2011) and Xiao et al. (2012) suggest the construction of these designs using conference matrices. The resulting DSD is always global optimum. This method is only applicable when the number of factors is even. This article introduces an algorithm for constructing DSDs for both even and odd numbers of factors using cyclic generators. We show that our algorithm can construct designs that are more efficient than those of Jones and Nachtsheim (2011) and that it can construct much larger designs.
\end{abstract}

AMS Subject Classification: 62K20.

Keywords: Coordinate exchange algorithm; D-efficiency; Incomplete block designs; Response surface designs; Screening designs.

\section{Introduction}

Engineers and scientists are more comfortable with to the notion that quantitative factors should have three levels and that designs for quantitative factors should be three-level designs. The most popular designs for screening experiments, however, are two-level designs (e.g., resolution III and IV fractional designs). Definitive screening designs (DSDs) are three-level designs introduced by Jones and Nachtsheim (2011) for studying $m$ quantitative factors with the following main desirable properties:

1. The design is mean orthogonal.

2. The number of required runs is $n=2 m+1$, that is, saturated for estimating the intercept, $m$ main effects, and $m$ quadratic effects.

3. Unlike resolution III designs, all main effects are orthogonal to all two-factor interactions.

4. Unlike resolution IV designs, two-factor interactions are not fully aliased with one another.

Received 7 May 2012; accepted 18 October 2012.

Address correspondence to: Nam-Ky Nguyen, International School \& Centre for High Performance Computing, Vietnam National University, HACINCO Student Village, 99 Nguy Nhu Kon Tum, Nhan Chinh, Thanh Xuan, Hanoi, Vietnam. Email: namnk@vnu.edu.vn 
5. All quadratic effects are orthogonal to main effects and not fully aliased with two-factor interactions.

The design matrix for a DSD can be written as

$$
\left(\begin{array}{r}
C \\
-C \\
\mathbf{0}
\end{array}\right)
$$

where $C$ is a constituent $m \times m(0, \pm 1)$-matrix with zero diagonal and $\mathbf{0}$ is a row vector of 0's. Stylianou (2011) and Xiao et al. (2012) point out that if we use a conference matrix order $m$ (which is identical to a weighing matrix of the same order and weight $m-1$ ) for $C$, then the DSD constructed by (1) is also orthogonal for main effects, that is, all main effects are orthogonal to one another. The Jones and Nachtsheim designs for $m=4,6,8$, and 10 have this property. The next section gives some information about conference matrices. Additional uses of conference matrices and weighing matrices in design of experiments can be found in Stylianou (2010) and Georgiou et al. (2013).

Since conference matrices of order $m$ are only available for even $m$, these matrices are only useful to construct DSDs with an even number of factors. For odd $m$, Xiao et al. (2012) point out that removing a row and the corresponding column of a conference matrix of order $m+1$ might not be a good idea as it might result in a singular matrix. This article introduces an algorithm that can generate the matrix $C$ in (1) of order $m$ for both even and odd numbers of factors $(m)$.

Section 2 gives the conference matrix in a nutshell. Section 3 explains the relationship between a conference matrix of order $m$ and a certain regular graph design (RGD). Section 4 describes an algorithm for constructing the $C$ matrix in (1). Section 5 gives the generating vectors for these matrices for both even and odd $m$ and compares the D-efficiencies of the DSDs constructed by the matrices with odd $m$ in this section and Jones and Nachtsheim's DSDs. Section 6 is our conclusion, which gives some directions for further studies.

\section{Conference Matrix in a Nutshell}

This section gives some information about conference matrices. More information about this type of matrix can be found, for example, in Section 6.1 of Ionin and Kharaghani (2007). A conference matrix $\mathbf{C}$ of order $m$ is an $m \times m(0, \pm 1)$-matrix with zero diagonal satisfying $\mathbf{C} \mathbf{C}^{\prime}=(m-1) I_{m \times m}$. The following is a conference matrix of order six (where + stands for +1 and - stands for -1$)$ :

$$
\begin{aligned}
& 0+++++ \\
& +0-++- \\
& +-0-++ \\
& ++-0-+ \\
& +++-0- \\
& +-++-0
\end{aligned}
$$

A conference matrix is said to be normalized if all entries in its first row and first column are 1 (except the $(1,1)$ entry, which is 0 ). If we remove the first row and the first column of a normalized conference matrix, we will be left with its core $\mathbf{S}$. It can be seen that the core of the conference matrix of order six just displayed is a circulant matrix generated by the vector $0-++-$. The core of every normalized conference matrix of order $m=4 t+$ $2(t \geq 1)$ is symmetric (i.e., $\left.\mathbf{S}=\mathbf{S}^{\prime}\right)$ and the core of every normalized conference matrix of order $m=4 t(t \geq 1)$ is skew-symmetric (i.e., $\mathbf{S}=-\mathbf{S}^{\prime}$ ). 
A skew-symmetric conference matrix of order $m=4 t$ can be constructed from a skewtype Hadamard matrix of the same order. In this article, we make use of this fact to construct several conference matrices from the generating vectors used to construct the PlackettBurman designs (Plackett and Burman (1946)). The following is a conference matrix of order 12 constructed from the Plackett-Burman design for 12 runs (generated by the generating array ++-+++---+- ) by replacing the +1 's in the diagonal of the latter by 0 's.

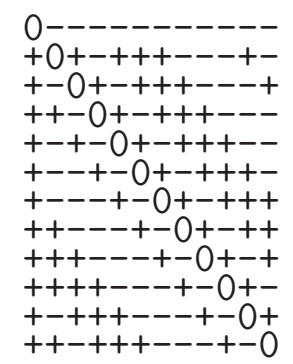

If $A$ is a $(0, \pm 1)$-matrix of order $m$ and $B$ a \pm 1 -matrix of the same order such that $A B=B A$ and $A A^{\prime}+B B^{\prime}=(2 m-1) I_{m \times m}$, then the following conference matrix of order $2 m$ can be constructed:

$$
\left(\begin{array}{cc}
A & B \\
B^{\prime} & -A^{\prime}
\end{array}\right)
$$

If $A$ is a circulant $(0, \pm 1)$-matrix of order $m$ and $B, C, D$ are circulant \pm 1 -matrices of the same order such that $A A^{\prime}+B B^{\prime}+C C^{\prime}+D D^{\prime}=(4 m-1) I_{m \times m}$, then the following conference matrix of order $4 m$ can be constructed:

$$
\left(\begin{array}{cccc}
A & B R & C R & D R \\
-B R & A & D^{\prime} R & -C^{\prime} R \\
-C R & -D^{\prime} R & A & B^{\prime} R \\
-D R & C^{\prime} R & -B^{\prime} R & A
\end{array}\right)
$$

Here $R$ is the back-diagonal identity matrix of order $m$, that is, the matrix whose elements $r_{i j}$ 's are all zeros except for those with $i+j=m+1$, which are ones, where $i, j=1, \ldots, m$. The following is a conference matrix of order 12 constructed using the Goethals-Seidel array of equation (3) with the circulant matrices $A, B, C$, and $D$ generated by the vectors $0+-,---,-++$ and -+ :

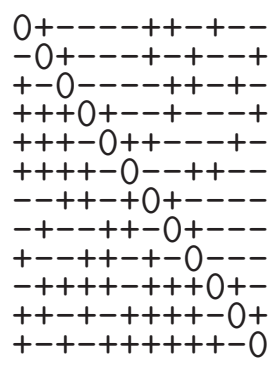


It is conjectured that conference matrix of order $m$ exists for all $m=4 t+2$ as long as $m-1$ is a sum of two squares. Of conference matrices of size 66 or less, the one of size 66 is unresolved and the ones of sizes 22,34 , and 58 do not exist.

\section{Relationship Between a Conference Matrix and an RGD}

There is a combinatorial relationship between certain combinatorial structures. Hedeyat and Wallis (1978) show that there is a relationship between a Hadamard matrix and several balanced incomplete block designs (BIBDs). Nguyen (1996) establishes a family of $E\left(s^{2}\right)$ optimal supersaturated designs from BIBDs. Greig et al. (2006) show that a conference matrix is related to a near-resolvable incomplete block design (IBD). In this section, we show that a conference matrix of order $m=4 t(t \geq 1)$ is related to certain BIBDs with $v=b=4 t-1$ and $k=r=2 t$ and a conference matrix of order $m=4 t+2$ is related to certain RGDs of size $v=b=4 t+1$ and $r=k=2 t+1$.

An IBD of size $(v, k, r)$ is an arrangement of $v$ treatments set out in $b$ blocks, each of size $k(<v)$ such that each treatment occurs in $r$ blocks where $v r=b k$ and no treatment occurs more than once in any block. If $v=b$ and $r=k$, the IBD is said to be symmetric. We avoid using this term for IBD in this article. The following is an IBD with $v=b=5$ and $r=k=3$ with blocks as columns:

\begin{tabular}{|l|l|l|l|l|}
\hline 0 & 1 & 2 & 3 & 4 \\
\hline 2 & 3 & 4 & 0 & 1 \\
\hline 3 & 4 & 0 & 1 & 2 \\
\hline
\end{tabular}

This IBD is also a cyclic IBD generated by the initial block (0 2 3). Cyclic IBDs are IBDs generated by the cyclic development of one or more suitably chosen initial blocks. Cyclic IBDs account for a large number of BIBDs in literature and can be easily constructed by computer. Associated with each IBD is its incidence matrix $N$ and concurrence matrix $N N^{\prime}=\left\{\lambda_{i j}\right\}$, where $\lambda_{i j}(i, j=1, \ldots, v)$ is the number of blocks in which treatment $i$ and $j$ both appear. Obviously, $\lambda_{i i}=r$. Since $\sum \lambda_{i j}$ is constant $(=v k r), \sum \lambda_{i j}^{2}$ is minimized if $\lambda_{i j}$ 's $(i \neq j)$ differ by at most 1 . IBDs with this property were called RGDs by John and Mitchell (1977), who conjectured that $D$-, $A$ - and $E$ - optimal IBDs are also RGDs. Thus, RGDs include BIBDs, that is, IBDs with $\lambda=r(k-1) /(v-1)\left(\right.$ since $\sum_{i \neq j} \lambda_{i j}=v k r-v r$ ) and IBDs with $\lambda_{1}=r(k-1) /(v-1)$ and $\lambda_{2}=\lambda_{1}+1$. The $N$ matrix of the IBD just described is

$$
\left(\begin{array}{lllll}
1 & 0 & 1 & 1 & 0 \\
0 & 1 & 0 & 1 & 1 \\
1 & 0 & 1 & 0 & 1 \\
1 & 1 & 0 & 1 & 0 \\
0 & 1 & 1 & 0 & 1
\end{array}\right)
$$

and its $N N^{\prime}$ matrix is

$$
\left(\begin{array}{lllll}
3 & 1 & 2 & 2 & 1 \\
1 & 3 & 1 & 2 & 2 \\
2 & 1 & 3 & 1 & 2 \\
2 & 2 & 1 & 3 & 1 \\
1 & 2 & 2 & 1 & 3
\end{array}\right)
$$


It is clear that this IBD is an RGD. Readers can find more information about IBDs in Raghavarao and Padgett (2005) or Nguyen and Blagoeva (2011). John and Williams (1995) give a very clear treatment of cyclic IBDs. Nguyen (1994) describes an algorithmic approach for IBD construction.

Let's assume that $m$ is even and consider an IBD of size $v=b=m-1$ and $r=k=$ $\frac{1}{2} m$. Let

$$
S=2 N-J-I
$$

where $J_{(m-1) \times(m-1)}$ is a matrix of 1's and $I_{(m-1) \times(m-1)}$ is an identity matrix. It can easily be shown that

$$
S S^{\prime}=4 N N^{\prime}-2\left(N+N^{\prime}\right)+(1-m) J+I
$$

First, consider the case $m=4 t$ and let's assume that we are able to construct a BIBD of size $v=b=4 t-1, k=r=2 t$ (and $\lambda=t$ ). If the $N$ matrix of this BIBD has the property $N+N^{\prime}=J+I$, it can be shown that $S S^{\prime}$ will have the diagonal elements being equal to $m-2$ and the off-diagonal element being equal to -1 . We use BIBD* to denote a BIBD with the property $N+N^{\prime}=J+I$. An example of a cyclic BIBD* with with $v=b=11$ and $r=k=6$ is the one generated by the initial block ( $\left.\begin{array}{lllll}0 & 1 & 3 & 4 & 5\end{array}\right)$.

Next, consider the case $m=4 t+2$ and assume that we are able to construct an RGD of size $v=b=4 t+1, k=r=2 t+1$ (and $\lambda_{1}=t$ and $\lambda_{2}=t+1$ ). If the $N$ matrix of this RGD has the property $N=N^{\prime}$, it can also be shown that $S S^{\prime}$ will have the diagonal elements being equal to $m-2$ and the off-diagonal elements being equal to -1 . We use RGD* to denote an RGD with the property $N=N^{\prime}$. An example of a cyclic RGD* with $v=b=5$ and $r=k=3$ is the one generated by the initial block (l $\left.\begin{array}{lll}0 & 2 & 3\end{array}\right)$ given at the beginning of this section.

Now, form the $C$ matrix as

$$
\left(\begin{array}{cc}
0 & \pm 1^{\prime} \\
\mathbf{1} & S
\end{array}\right)
$$

where 1 is a $(m-1) \times 1$ column vector of 1 's and $S$ is the matrix in (4) formed by the $N$ matrix of either the BIBD* or RGD*. It can be verified that $C C^{\prime}=(m-1) I_{m \times m}$, that is, $C$ is a conference matrix. The conference matrix of order six displayed in section 2 is in fact derived from the cyclic RGD* with $v=b=5$ and $r=k=3$ generated by the initial block ( $\left.\begin{array}{lll}0 & 2 & 3\end{array}\right)$ mentioned earlier. Similarly, the first conference matrix of order 12 displayed in section 2 can be constructed from the cyclic BIBD* with $v=b=11$ and $r=k=6$ generated by the initial block ( $0 \begin{array}{lllll}0 & 1 & 3 & 4 & 5\end{array}$ ) mentioned earlier.

While the RGD approach is efficient, it does not guarantee the best $C$ when $m$ is odd.

\section{A General Algorithm for Constructing an $m \times m(0, \pm 1)$-Matrix With Zero Diagonal}

The previous section gives two examples of cyclic IBDs. To construct the $C$ matrix in equation (1), our approach is to associate this matrix with a cyclic IBD. For $m=4 t$, we associate this matrix with a BIBD* of size $v=4 t-1$ and $k=r=2 t$. For $m=4 t+2$, we associate this matrix with an $\mathrm{RGD}^{*}$ of size $v=b=4 t+1$ and $k=r=2 t+1$. In the following paragraph, we use IBD* to denote any IBD of size $v=b=4 t-1$ and $k=r=2 t$ 
with the property $N+N^{\prime}=J+I$ or any IBD of size $v=b=4 t+1$ and $k=r=2 t+1$ with the property $N=N^{\prime}$. Now, the steps for even $m$ are:

1. Generate an initial block for a cyclic IBD of with the appropriate size. The initial block of this cylic IBD should always contain treatment 0 .

2. Use the coordinate exchange algorithm (CEA) of Meyer and Nachtsheim (1995) with treatments other than treatment 0 in the initial block to force this cyclic IBD to become a cyclic IBD*.

3. Use the mentioned CEA with treatments other than treatment 0 in the initial block to force this cyclic IBD* to become a cyclic BIBD* if $m=4 t$ or a cyclic RGD* if $m=$ $4 t+2$.

4. Use equations (4) and (5) to construct the $C$ matrix from the $N$ matrix of the obtained cyclic BIBD* or RGD* in step 3.

For odd $m$, we have two approaches. In the first approach, we aim for a cyclic RGD of size $v=b=m-1, r=k=\frac{1}{2} v$ and use equations (4) and (5) to construct $C$. In the second approach, we aim for a cyclic RGD of size $v=b=m$ and suitable $k(=r)$. The core of the $C$ matrix is then computed as $2 N-J_{m \times m}-I_{m \times m}$. In either approach, among a large number of generated $C$ matrices, the one with the largest $\left|C^{\prime} C\right|$ (determinant of $C^{\prime} C$ ) is selected.

Our algorithm is reasonably fast. It requires just a couple of seconds to generate 10,000 $12 \times 12 C$ matrices on an Intel Core 2 Duo $2.60 \mathrm{GHz}$ laptop. Out of these 10,000 matrices, 1,250 are conference matrices. Note that the Jones and Nachtsheim CEA algorithm fails to find a conference matrix for this order. Our algorithm, however, requires about 20 seconds on the same laptop to generate $10,00048 \times 48 C$ matrices. Out of these 10,000 matrices, just two are conference matrices.

\section{Results and Discussion}

Table 1 provides the generating vectors for the $C$ matrices in equation (1) with even $m \leq 50$. Unless stated otherwise, these vectors are used to construct the core $S$ in equation (5). Some of these matrices for $m=4 t$ are obtained from the generating vector of the PlackettBurman design by switching the first element of its generating vector from +1 to 0 . The $C$ matrices for $m=16$ and 40 are constructed by equation (2) where $A$ is the conference matrix of the order $\frac{1}{2} m$ constructed by the generating vector in Table 1 and $B=A+I$.

The $C$ matrices for $m=10,22,26,34$, and 50 are also constructed by equation (2) where $A$ and $B$ are two circulant matrices generated by the vectors in Table 1 . The generating vectors for these five designs are constructed by trial and error.

The $C$ matrices for $m=28$ and 36 are constructed by equation (3) where $A, B, C$, and $D$ are four circulant matrices generated by the vectors in Table 1 . The method of constructing the $C$ matrix for $m=46$ is described in Seberry and Whiteman (1988).

With the exception of $m=22$ and 34, the $C$ matrices generated by the vectors in Table 1 for other $m$ are conference matrices. We can measure the goodness of a DSD that is not constructed by a conference matrix by studying its D-efficiency. Let $X_{(2 m+1) \times(2 m+1)}$ be the expanded design matrix, which includes the mean, $m$ main effect, and $m$ quadratic effect terms. Since $|X|=|C| \times 2^{m}(m-1)$ (cf. Section 1 of Appendix A of Xiao et al. 2012), the D-efficiency of a DSD can be calculated as $\left(\left|C^{\prime} C\right| /(m-1)^{m}\right)^{1 /(2 m+1)}$. This is the relative D-efficiency between this DSD and a conference matrix-based DSD for the same size assuming that the latter exists. The D-efficiencies of our designs for 22 and 34 factors are 


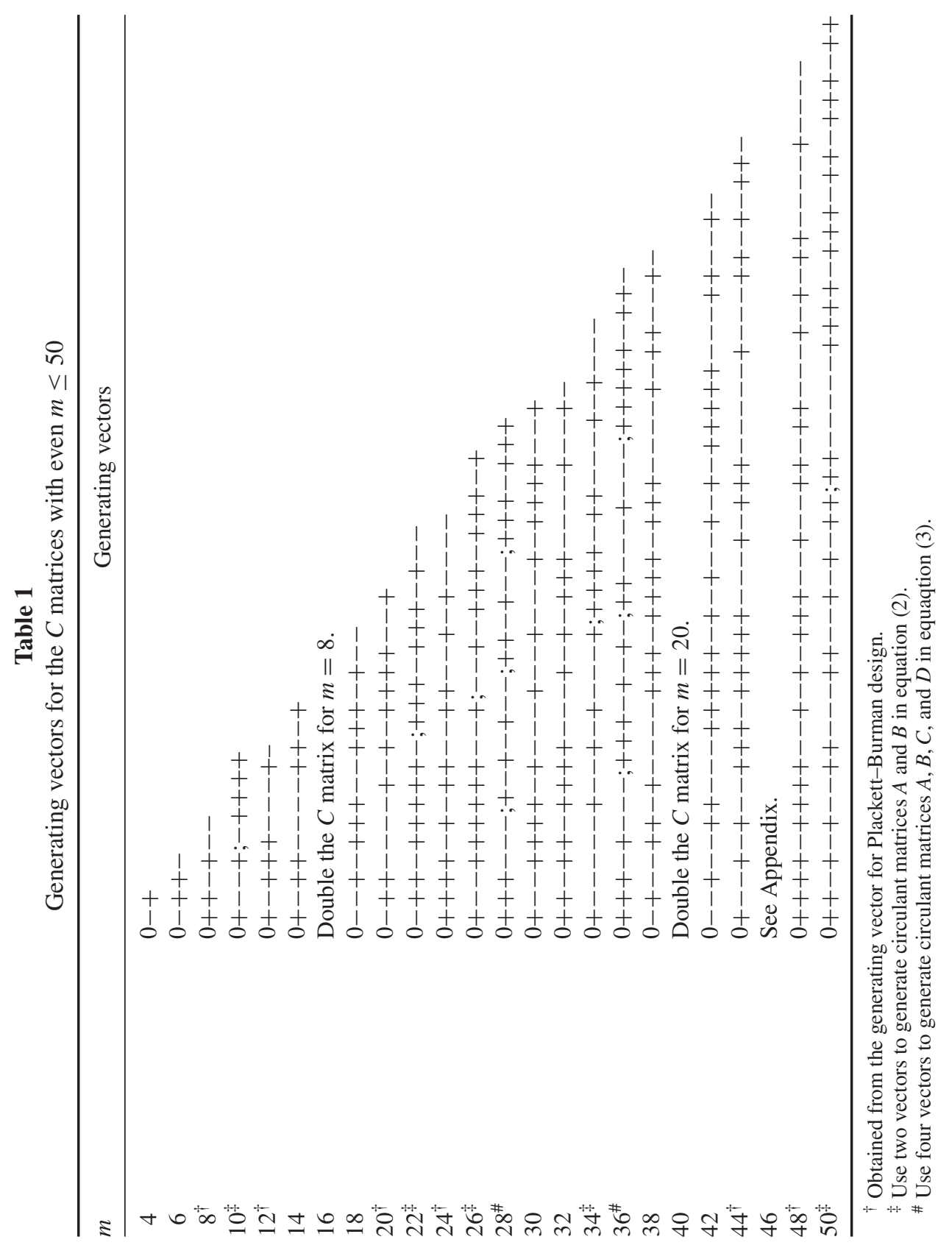


$99.55 \%$ and $99.82 \%$, respectively. Note that the D-efficiency of the JN design for 22 factors is $96.84 \%$.

Table 2 gives the generating vectors for matrices $C$ with odd $m \leq 49$. Arrays for designs marked with $(\dagger)$ are associated with an initial block of an RGD or IBD with $v=b=m-1$ and $r=k=\frac{1}{2} v$. The circulant matrices generated by these vectors are used to construct the core matrix $S$ in equation (5). The remaining generating vectors are associated with an initial block of an $\operatorname{RGD}$ or IBD size $(v, k, r)=(m, k, k)$ for a suitable value of $k$.

Table 3 compares the D-efficiencies of JN designs and new designs. This table shows that with one exception for $m=11$, all new designs are better than Jones and Nachtsheim designs and that our algorithm is more suitable for constructing large designs.

\section{Concluding Remarks}

In the previous sections, we have discussed the construction of the $C$ matrix in equation (1). Each column of $C$ so far contains a single 0 value. This means that each factor in our DSDs will only have $m-1$ runs at high level (or +1$), m-1$ runs at low level (or -1 ) and three runs at mid-level (or 0). Certainly, this DSD will not be a good choice of a design matrix if the mid-levels of several factors happen to be the desirable levels. Stylianou (2011) discusses the possibility of using weighing matrices of order $m$ and weight less that $m-1$ as the $C$ matrix in equation (1). In this case, each factor in a DSD will have more than three mid-levels.

The following is an alternative $C$ matrix for a DSD with 22 factors. This matrix is generated by equation (2) using two circulant arrays with generating vectors $0+-----++-$ and $0+-+--+-+++$. Readers can verify that it is a weighing matrix of order 22 and weight 20 (i.e., $C C^{\prime}=20 I_{22 \times 22}$ ).

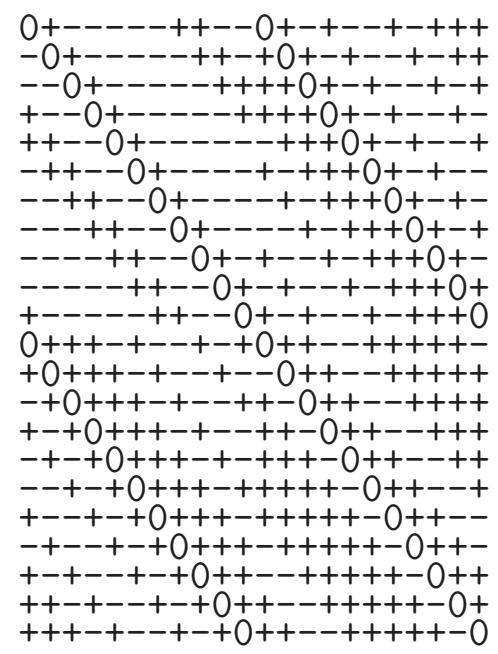

Reader can also verify that the matrix generated by equation (2) using the two circulant arrays with generating vectors $0-++--++-----+-+-$ and $0------+-++$ -+--++ is a weighing matrix of the order 34 and weight 32 . The two $C$ matrices in this section can be used as good alternatives to the ones for 22 and 34 factors in Table 1. These 


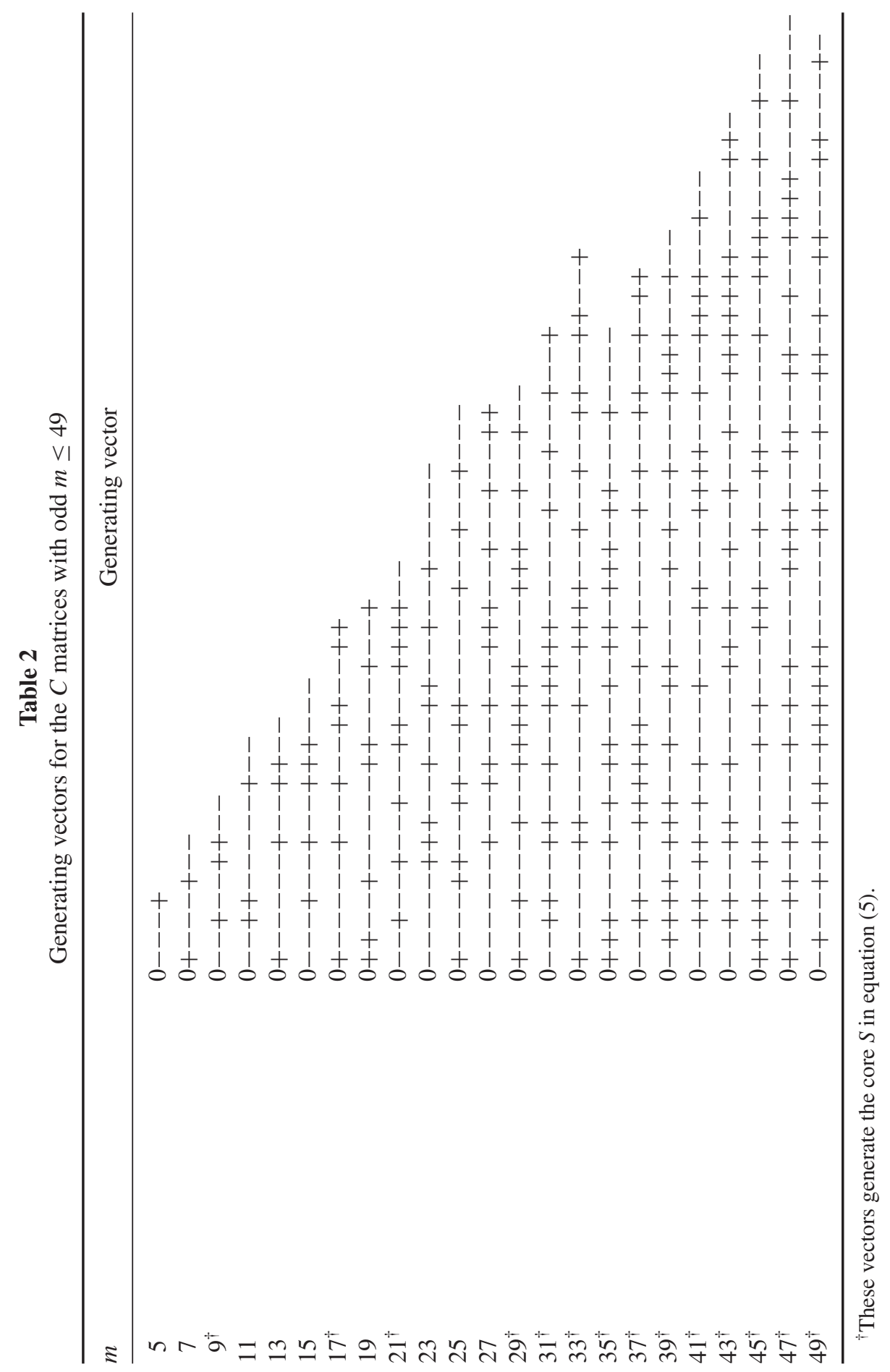


Table 3

Comparing D-efficiencies (\%) of Jones and Nachtsheim (JN) designs and new designs for odd $m$

\begin{tabular}{|c|c|c|c|}
\hline$m$ & JN designs & New designs & Relative ratio $(\%) \dagger$ \\
\hline $5^{\ddagger}$ & 93.41 & 93.41 & 100.00 \\
\hline $7 \ddagger$ & 96.15 & 96.15 & 100.00 \\
\hline $9 \ddagger$ & 97.10 & 97.10 & 100.00 \\
\hline $11^{\ddagger}$ & 97.66 & 97.49 & 99.83 \\
\hline $13^{\ddagger}$ & 97.82 & 97.94 & 100.12 \\
\hline $15^{\ddagger}$ & 97.66 & 98.48 & 100.84 \\
\hline 17 & 97.34 & 97.49 & 100.16 \\
\hline $19:$ & 96.86 & 98.51 & 101.70 \\
\hline 21 & 96.85 & 98.23 & 101.43 \\
\hline $23^{\ddagger}$ & 96.83 & 98.97 & 102.21 \\
\hline 25 & 96.72 & 98.12 & 101.45 \\
\hline 27 & 96.74 & 98.54 & 101.86 \\
\hline 29 & 96.62 & 99.20 & 102.67 \\
\hline $31^{\ddagger}$ & - & 99.13 & - \\
\hline 33 & - & 98.61 & - \\
\hline 35 & - & 98.34 & - \\
\hline 37 & - & 98.66 & - \\
\hline 39 & - & 98.75 & - \\
\hline 41 & - & 98.80 & - \\
\hline 43 & - & 99.17 & - \\
\hline 45 & - & 98.66 & - \\
\hline 47 & - & 98.65 & - \\
\hline 49 & - & 98.66 & - \\
\hline
\end{tabular}

D-efficiency of new design/D-efficiency of JN design.

$\stackrel{\$}{*}$ off-diagonal elements of $C C^{\prime}$ of new design equal \pm 1 .

two matrices, like the ones of order 10, 22, 26, and 34 in Table 1, were generated by trial and error. A good algorithm to generate these matrices and the relationship between them and other combinational structures, like IBDs, merit further studies.

\section{Acknowledgment}

The authors are grateful to the referee for helpful comments and corrections of the first draft. The first author wishes to dedicate this paper to Nye John, a mentor and friend.

\section{References}

Georgiou S. D., S. Stylianou, and M. Aggarwal. 2013. Efficient three-level screening designs using weighing matrices. Statistics, 1-19.

Greig, M., H. Haanp, and P. Kaski. 2006. On the coexistence of conference matrices and near resolvable $2-(2 k+1, k, k-1)$ designs. Combinatorial Theory Ser. A 113, 703-711.

Hedeyat, A., W. D. Wallis. 1978. Hadamard matrices and their applications. Ann. Stat. 6, 1184-1238. 
Ionin Y. J. and H. Kharaghani. 2007. Balanced generalized weighing matrices and conference matrices. In Handbook of combinatorial designs, 2nd ed., edi. J.H. Colbourn, 306-313. Boca Raton, FL, CRC Press.

John, J. A., and T. J. Mitchell. 1977. Optimal incomplete block designs. J. of the Royal Statistical Society, Ser. B, 39, 39-43.

John J. A. and E. R. Williams. 1995. Cyclic Designs and Computer-Generated Designs. London, Chapman \& Hall.

Jones, B., and C. J. Nachtsheim. 2011. A class of three levels designs for definitive screening in the presence of second order effects. J. Quality Technolo., 43, 1-15.

Meyer, R. K., and C. J. Nachtsheim. 1995. The co-ordinate exchange algorithm for constructing exact optimal experimental designs. Technometrics, 37, 60-69.

Nguyen, N.-K. 1994. Construction of optimal block designs by computer. Technometrics, 36, 300-307.

Nguyen, N.-K. 1996. An algorithmic approach to constructing supersaturated designs. Technometrics, $38,69-73$.

Nguyen, N.-K., and K. L. Blagoeva. 2011. Incomplete block designs. In Encyclopedia of statistical science, edi. L. Miodrag, 653-655. Springer.

Plackett, R. L., and J. P. Burman. 1946. The design of optimum multifactorial experiments. Biometrica, 33, 305-325.

Raghavarao, D., and L. V. Padgett. 2005. Block designs: Analysis, combinatorics and applications. Singapore, World Scientific.

Seberry, J., and L. Whiteman. 1988 New Hadamard matrices and conference matrices obtained via Mathon's construction. Graphs Combinatorics, 4, 355-377.

Stylianou, S. 2010. Foldover conference designs for screening experiments. Communi. Stat. Theory Methods, 39, 1776-1784.

Stylianou, S. 2011. Three-level screening designs applicable to models with second order terms. Paper presented at the International Conference on Design of Experiments (ICODOE-2011). May 10-13. Department of Mathematical Sciences, University of Memphis, Memphis, TN.

Xiao, L., D. K. J. Lin, and F. Bai. 2012. Constructing definitive screening designs using conference matrices. J. Quality Technol., 44, 2-8. 
Appendix: A Conference Matrix of Order 46

$0+++++++++++++++++++++++++++++++++++++++++++++$ +0 ++-+---+++--+++-+--+--+--+---+++---+-+-++++++0 $0+--++---+++-+++---+--+--+------+++++-+-+-++$ ++ $+0+---+-+-+++--++--+--+--++++-------++++-+-+$ +- - $+0++-+--+++-+++-+--+--+-----+++----++++-+-+$ ++-- $+0+--++-+++--+++--+--+--------++++-+-++++-$ +- $+-++0+--++--+++-++--+--+--+++------++-+-+-++$ +- +- - $+0+++-+++--++-+--+--+----+++---++-+-+-++$ +- - + $+--+0+++--+++-+-+--+--+-------+++-++++-+-+$ ++-- - +- + $+0-+++-+++--+--+--+-+++------+-+-++++-$ ++- +- ++++-0 $0+-+---+++--+++-+--+--+--+---+++---$ +++- $+-+-+++0+--++---+++-+++---+--+--+------+++$

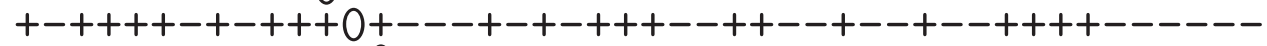
+- ++++- +- +- - $0+0+-+--+++-+++-+--+--+-----+++---$ ++- +- ++++- $+--+0+--++-+++--+++--+--+--------+++$ +++-+-+- ++- +- + $+0+--++--+++-++--+--+--+++------$ +++- +- +- ++- +- - $+0+++-+++--++-+--+--+----+++---$ +- ++++-+-+-- ++-- $0+++--+++-+-+--+--+-------+++$ ++- $+-++++-+---+-++0-+++-+++--+--+--+-+++------$ +- - $+++---+-+-++++-0++-+---+++--+++-+--+--+--+$ +----- $+++++-+-+-+++0+--++---+++-+++---+--+--+$ ++++------ $++++-+-+++0+---+-+-+++--++--+--+--+$ +-- $+++----++++-+-+--+0++-+--+++-+++-+--+--+--$ +------++++- +- ++++-+-- $0+0+-++-+++--+++--+--+--$ ++++----- $++-+-+-++-+-++0+--++--+++-++--+--+--$

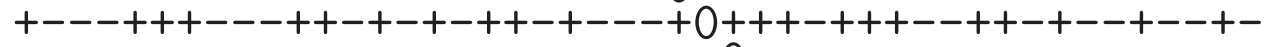
+- - - - - +++- ++++- +- +- - ++- $+0+++--+++-+-+--+--+-$ ++t+-- - - - +- $+-++++-+---+-++0-+++-+++--+--+--+-$ +- - +- $+--+---+++---+-+-++++-0++-+---+++--+++-+$

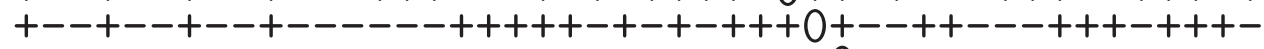
+--+--+--++++------ $++++-+-+++0+---+-+-+++--++$ ++- $+--+-----+++----++++-+-+--+0++-+--+++-+++-$ ++- - +- - +- - - - - - - + +++- $+-++++-+--+0+--++-+++--++$ ++--+--+-- +++----- $++-+-+-++-+-++0+--++--+++-+$ +- +- - +- - +- - - $+++---++-+-+-++-+---+0+++-+++--++$ +- +- - +- - +- - - - - - + + $+-++++-+-+--++--+0+++--+++-+$

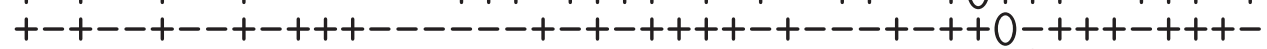

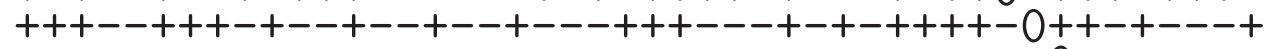

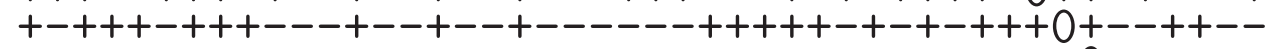
++- +++- $++--+--+--++++-------++++-+-+++0+---+-$

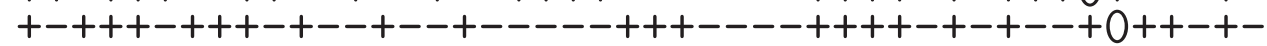
++- +++- $+++--+--+--------++++-+-++++-+--+0+--+$ +++- $+++-++--+--+--+++------++-+-+-++-+-++0+--$ ++- +++- $++-+--+--+----+++---++-+-+-++-+---+0++$ t++-- $+++-+-+--+--+-------+++-++++-+-+--++--+0+$ +-+++-+++--+--+--+-+++------+-+-++++-+---+- + 0 